\title{
The importance of the precorneal tear film for the quality of optical imaging
}

\section{Department of} Ophthalmology of the Paracelsus Institute, Bad Hall, Austria G Rieger

Correspondence to: Prim Dr G Rieger, Leiter der Augenabteilung des Paracelsus-Institutes, A-4540 Bad Hall, Austria.

Accepted for publication 8 August 1991

\begin{abstract}
The optical function of the eye critically depends on the presence of an intact precorneal tear film. Abnormalities of the tear film such as those occurring in the dry eye syndrome or in otherwise irritated eyes may interfere with vision. In 30 patients with dry eyes artificial tears were found to produce stastistically significant improvement of vision $(\mathbf{p}<0.001)$ while no significant changes were seen in the untreated partner eyes. Static perimetry in another 30 patients using the 'macular programme' of the automatic Humphrey 620 perimeter showed artificial tears to increase mean thresholds significantly $(\mathbf{p}<0.001)$ whereas no significant differences were seen in the untreated partner eyes.
\end{abstract}

With a refractive power of 49 dioptres the corneal surface is the highest refractive optical interface in the human eye. ${ }^{1}$ However it needs continuous and homogeneous wetting for optimal quality of the optical image and function of the eye. Wetting is provided by the three layers of the precorneal tear film which guarantee a smooth and even surface. Thus the tear film is a 'polish' of the most important refractive medium - the cornea. ${ }^{2}$

Apart from preventing dryness and infection and protecting the transparency of the cornea by its hyperosmolarity the tear film is essential for ensuring an optimal optical function of the eyes. Like the cornea and the crystalline lens it is among the important optically relevant structures of the refractive media. ${ }^{3}$ If unstable it impairs vision. ${ }^{3}$

Appreciable variations of visual acuity in patients with dry eyes and the reported occurrence of (pseudo) scotomas seen on perimetry prompted us to investigate the effect of tear film stability on the quality of optical images and vision.

\section{Material and methods}

Thirty patients without ocular abnormalities (18 females, 12 males; worst vision $0 \cdot 8$ ) aged between 42 and 78 years (mean age $60 \cdot 4$ years) presenting with dry eyes were investigated. Dry eyes were diagnosed on the basis of subjective symptoms $-6 \mathrm{~mm}$ wetting or less in the Schirmer test in local anaesthesia (means $2.83 \mathrm{~mm} \mathrm{OD}$ and $2.37 \mathrm{~mm}$ OS with test strips in OD first) and a break-up-time (BUT) of less than $10 \mathrm{~s}$ (mean $3.0 \mathrm{~s}$ ). Under standardised conditions (DIN 58 220 ) optimally corrected vision was evaluated separately for the two eyes 1 day after the Schirmer test and the determination of the BUT. One drop of a $2 \%$ poly(vinylpyrrolidone) solution was applied to the right eye and vision was again tested 5 minutes later. Visual acuities before and after tear replacement were compared. Analyses were based on the difference in the number of correctly read optotypes. The untreated left eyes served as controls.

In another group of 30 patients with dry eyes (19 females, 11 males; worst vision, 0.8) aged between 43 and 76 years (mean age 61 years) perimetric thresholds were determined 1 day after keratoconjunctivitis sicca had been diagnosed. Using the 'macular programme' of the Humphrey 620 computerised perimeter data were first obtained for the right eye. After instilling artificial tears into the right eye thresholds were determined for the left eye. Then fields were again tested for both eyes with the right eye being examined first. Thresholds in $\mathrm{dB}-$ that is, means of three readings, were evaluated. The sum total of all 16 measuring points before tear replacement was compared with that after tear replacement. Statistical analysis was performed with Wilcoxon's matched pair test. ${ }^{5}$ The untreated left eyes served as controls.

\section{Results}

In the group of patients with tear replacement in OD 25 of the 30 eyes (83\%) showed improved visual acuity by 1 to 4 optotypes (mean $+2 \cdot 6$ optotypes). In five eyes (17\%) visual acuity was unchanged.

Of the 30 left eyes which served as untreated controls $17(56 \%)$ showed no statistically significant improvement ( 1 to 4 optotypes (mean $+1 \cdot 6$ optotypes)). Six eyes (20\%) were unchanged. In seven eyes acuity had slightly deteriorated by 1 to 2 optotypes (mean -1.4 optotypes).

Statistical analysis using Wilcoxons's matched pair test showed a mean acuity gain of +2.33 optotypes after tear replacement. The difference was highly statistically significant (significance level $99 \cdot 999 \%$ ).

In the control group a mean gain of +0.54 optotypes was seen, but the difference was not statistically significant (significance level 92.313\%) (Fig 1).

Macular thresholds determined by static computerised perimetry showed a similar pattern. Of the 30 patients with tear replacement in OD 20 $(66.66 \%)$ were found to have gained between +1 and $+39 \mathrm{~dB}$ (mean $+11.75 \mathrm{~dB}$ ), while 10 patients $(33 \cdot 3 \%)$ had lost between -1 and -18 $\mathrm{dB}($ mean $-8 \cdot 3 \mathrm{~dB})$.

In the untreated controls ( 30 left eyes) thresholds had increased by +1 to $+17 \mathrm{~dB}$ (mean +6 $\mathrm{dB}$ ) in 12 eyes ( $40 \%$ ) while 18 eyes $(60 \%)$ had lost between -2 and $-17 \mathrm{~dB}$ (mean $-7 \cdot 77 \mathrm{~dB}$ ).

Statistical analysis using Wilcoxon's matched pair test showed mean threshold gains of $+0 \cdot 31$ 
Figure 1 Visual acuity examinations (number of correctly read optotypes of the obtained line of acuity) before (column 1) and after treatment with artificial tear fluid (column 2) and controls without artificial tear fluid (column 3, column 4:5 minutes later). Statistical significance (Wilcoxon's paired test $): \star=9.999 \%$ and $\star \star 92 \cdot 813 \%$ compared with examination before rest $(\mathrm{n}=30)$

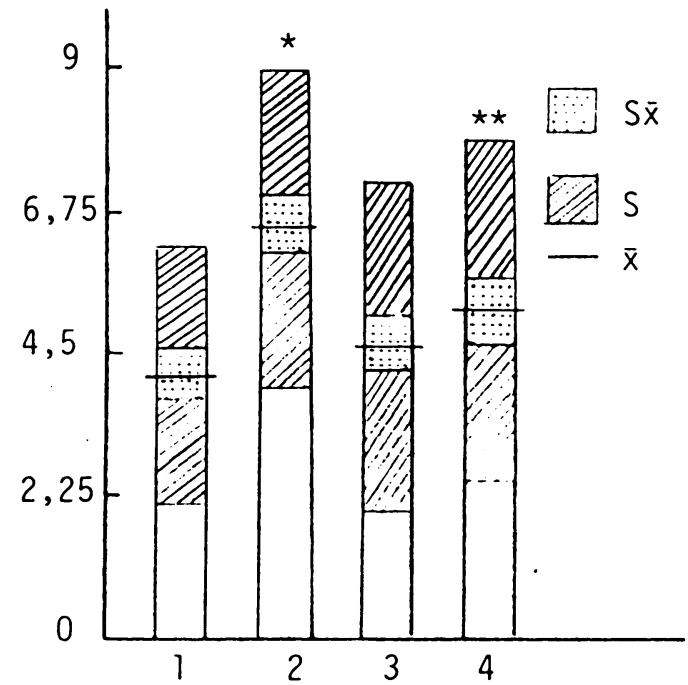

$\mathrm{dB}$ after tear replacement. The difference was again highly statistically significant (significance level $99 \cdot 99 \%$ ).

In the untreated controls mean thresholds had decreased by $-0.11 \mathrm{~dB}$. The difference was not statistically significant (significance level 94.54\%) (Table 1).

\section{Discussion}

Our studies underscore the significance of an intact and stable precorneal tear film for the quality of optical images and for the optical function of the eye. In patients with dry eyes tear replacement apparently had a beneficial effect on the physical and chemical properties of the precorneal tear film and on the corneal surface. This was reflected in a statistically significant improvement of visual acuity and of threshold readings on static perimetry. The reduced threshold in visual field testing by itself does not prove that a poor tear film causes pseudoscotomas. However the improvement of threshold readings
Table 1 Wilcoxon's paired test of the differences $(d B)$ between the first and the second test of the static computerised perimetry (macula programme)

\begin{tabular}{llll}
\hline & $\begin{array}{l}\text { Difference } \\
(\mathbf{n})\end{array}$ & Mean & $\begin{array}{l}\text { Significance } \\
\text { level }\end{array}$ \\
\hline Right eye $^{\star}$ & 480 & +0.31 & $>99.99 \%$ \\
Left eye $^{\star}$ & 480 & -0.11 & $94.54 \%$ \\
\hline
\end{tabular}

$\star$ Right eye: treatment with artificial tear fluid Left eye: without treatment.

on static perimetry after instilling artificial tears may suggest this possibility. In patients with dry eyes undergoing examinations, that is perimetry as well as acuity tests, this should be allowed for. Focusing during examinations with fewer blinks but also a reduced blinking rate as seen while watching $T V$, reading, in screen-workers, etc further reduces the stability of the tear film in patients with dry eyes so that acuities and thresholds may appear worse than they actually are. Even in patients with normal tear function (including children!) but otherwise irritated eyes (contact lens wearers, in air-conditioned rooms, in airplanes, trains, etc) the stability of the tear film may be considerably disturbed. In patients with reduced tear film stability certain examinations such as acuity tests and perimetry should therefore be preceded by the instillation of artificial tears.

1 Jaeger W, Blassmann $\mathrm{K}$. Bedeutung des präkornealen Films für die Qualität der optischen Abbildung. In: MeyerSchwickerath G, Ulrich K, eds. Moderne Probleme der Erkrankungen der Lider und des Tränenapparates. Bücherei des Augenarztes. Stuttgart: Enke Verlag, 1978; 75: 204-19.

2 Jaeger W. Der präkorneale Film und seine Bedeutung für die Therapie des 'trockenen Auges'. In: Hanselmayer $\mathrm{H}$, eds. Neue Erkenntnisse über Erkrankungen der Tränenwege: Neue Erkenntnisse über Erkrankungen der Tränenwege: Büchere $40-53$.

3 Gomez da luz R, Ferraz de Oliveira LN. The dioptric power of the tear film. In: Ferraz de Oliveria LN, ed. Ophthalmology Today. Proc 8th Congr Eur Soc Ophthalmol, Lisbon, 16-20 May 1988. Amsterdam: Excerpta Med, 1988: 499-500

4 Heilig P. Personal communication, 1989.

5 Der Wilcoxon-Test für Paardifferenzen (Wilcoxon matched pairs signed ranktest). In: Sachs L, ed. Angewandte Statistik, 6th edition. Berlin, Heidelberg, New York, Tokyo: Springer Verlag, 1984: 244-6. 